\title{
Elevated Nitrate Simplifies Microbial Community Compositions and Interactions in Sulfide-rich River Sediments
}

\author{
Enze Li $^{1}$, TONGCHU DENG ${ }^{1}$, LEI YAN ${ }^{1}$, Jizhong Zhou ${ }^{2}$, Zhili He ${ }^{3}$, Ye Deng ${ }^{4}$, and \\ Meiying $\mathrm{Xu}^{1}$ \\ ${ }^{1}$ Guangdong Institute of Microbiology \\ ${ }^{2}$ University of Oklahoma \\ ${ }^{3}$ Sun Yat-Sen University \\ ${ }^{4}$ Chinese Academy of Sciences
}

April 28, 2020

\begin{abstract}
In river sediments, nitrate is a more preferable electron sink for microbial respiration than oxygen, for its good solubility and high redox potential. Nitrate amendment has been used to accelerate oxidation of pollutants but very few studies focused on microbial dynamics during bioremediation. Here we simulated a nitrate surge and monitored the microbial responses in a sulfide-rich river sediment. Original communities were found dominated by methanogens and syntrophic bacteria, yet nitrate input enriched two chemolithotrophic denitrifiers Thiobacillus and Luteimonas, in spite of abundant organic carbons there. This led to simplified community composition, function and interactive networks. Similarly, serial dilutions of sediments found that Thiobacillus thiophilu s dominated 18/30 communities because of its simultaneous nitrate reduction and sulfide oxidation. Interestingly, syntrophic bacteria and archaea performing interspecies cross-feeding rather than the dominant denitrifiers were sustaining microbial interactions. Therefore, environment perturbations that inhibit native auxotrophs will very likely disrupt original microbial interactions.
\end{abstract}

\section{Author contribution}

All authors conceived the study; MX and JG designed the experiment; TD and LY performed the experiment; EL and TD analyzed data; MX, EL, ZH, YD and JZ wrote the manuscript. All authors revised the manuscript.

\section{Data Availability}

Raw Illumina reads of the $16 \mathrm{~S}$ ribosomal RNA gene used to track community successions and to construct microbial interactive networks are available in the NCBI SRA. BioProject: Ronggui-sediment, Accession: PRJNA526396 (https://www.ncbi.nlm.nih.gov//sra?term=PRJNA526396).

\section{Conflict of Interest}

The authors declare no competing interests.

\section{Novelty statement}

Nitrate amendment has been extensively used in microbial bioremediation to help exhaust the reductant pollutants in anaerobic habitats, but very few studies focused on the microbial dynamics. Previously we treated sulfide-rich river sediments for a week and identified enriched functional genes involved in nitrogen and sulfur cycles. In this study, we extended the observation to a month and focused on how nitrate amendment affected microbial community assembly. 
The first finding was as predicted that elevated nitrate led to a taxonomic convergence at denitrifiers (Thiobacillus andLuteimonas), and a functional convergence at denitrification and sulfide oxidation. The resulting communities had significantly decreased biodiversity and became highly fragile to environmental perturbations. With so abundant organic carbon present in subject sediments however, the most enriched denitrifiers were obligate chemolithotrophs. In addition, when looking into the microbial interactive networks, the keystone taxa consisted of neither the most abundant taxa nor the best metabolic competitors, but those performing interspecies cross-feeding, e.g. syntrophic bacteria and methanogens.

\section{Introduction}

Microorganisms in nature co-exit as communities, and species interactions are essential to stabilize their composition and functionality (Donaldson et al. 2016). Members of a community may interact bidirectionally or unidirectionally, and microscale communities frequently assemble and disassemble by migration, attachment, and detachment from surfaces or cells, leading to mutualism, parasitism or competition among them (Cordero \& Datta 2016).

Environmental fluctuations are altering the biodiversity, which in turn is a key driver of biogeochemical processes. Reactive nitrogen (Nr, all nitrogen species other than N2) is widely utilized by plants and algae (Chen et al. 2011) but may cause ecological issues if too abundant. In the past few decades, vast quantities of $\mathrm{Nr}$ have been produced to meet the increasing demand for food and energy (Lutz et al. 2001). Many of them can be nitrified to highly mobile nitrate and are leached into rivers, leading to eutrophication and seasonal hypoxia (McIsaac et al. 2001; Fowler et al.2013). Rivers were reported to convert (50 \pm 20$) \%$ of $\mathrm{Nr}$ inputs from adjacent ecosystems into N2 while transporting the other half to coastal water (Xia et al. 2018). River sediments contributed a significant part of total nitrogen transformation.

In subsurface or deeper sediments where oxygen cannot penetrate, nitrate ranks the best alternative electron acceptor in association with anaerobic oxidation of organic matters, sulfide and ferrous iron (Burgin \& Hamilton 2007). It was reported that nitrate reduction accounted for 10 to $40 \%$ nitrate removal in the sediments of Michigan wetlands, lakes and creeks (Brunet \& GarciaGil 1996; Burgin \& Hamilton 2008). A number of studies have obtained positive effects of nitrate input into the sulfide-rich river sediments by stimulating denitrifiers, autotrophic sulfide oxidizers and Fe(II) oxidizers (He et al. 2017; Heet al. 2018). Our previous study also identified an enrichment of functional genes involved in nitrogen (e.g. nir , nif , nor and nas ) and sulfur (e.g. sox, dsr ,apr and sir ) cycles when native microorganisms were exposed to elevated nitrate for 6 days (Xu et al. 2014).

In an ecological view however, external nitrate injection is considered an environmental perturbation, and how it shapes microbial communities, especially in a long run, has not yet been studied. Next-generation sequencing (NGS) provides extensive inferences about microbial community structure and functional profile, which are high-throughput, culture-independent, and reproducible, but are far less convincing than solid experimental data. In 2012, an approach for identifying new strains, microbial interactions and functions by culturing not a single isolate but the entire community with serial dilution was proposed (Lagier et al. 2012; Lagier et al. 2016). This approach has been so far explored in human gut and animal rumen microbiomes (Zehavi et al. 2018). Considering the key role of nitrate in biosphere and the complexity of microbial network involving in nitrogen cycling, the combination of NGS and community enrichment could be the promising strategy.

In this study, we hypothesized (i) that elevated nitrate would enrich native denitrifiers in association with a suppression of methanogens and sulfate reducers, resulting in much specialized sediment communities; (ii) that the overall community functionality would gradually converge at nitrate reduction coupled with sulfide oxidation; and (iii) that the microbial interactive networks would be remodeled due to the rise of denitrifiers and their metabolic specializations. Excess nitrate was injected to river sediments in the Pearl River Delta, which was rich in organic carbon and sulfide, to simulate a natural nitrate surge. Community successions and physio-chemical conditions were monitored for 32 days. In addition to 16S rRNA gene amplicon sequencing, community enrichments were cultured by serial dilution to isolate strains of sulfur-oxidizing denitrifiers, and 
to match the results to those of NGS. Our work describes new entry points for the undefined microbial mechanisms of nitrate-induced biogeochemical cycling in river sediments.

\section{Results}

\section{Nitrate input induced AVS and Fe(II) oxidation}

Five sampling timepoints divided the incubation into four stages. Each stage of the nitrate group had distinct physiochemical features. On Day 4, nitrate was consumed by $13.8 \%$ (from 7.74 to $6.82 \mathrm{mM}, P<0.001$ ) but little nitrite $(<0.01 \mathrm{mM})$ was produced (Figure S1A). Similarly, acid volatile sulfide (AVS) was consumed by $19.3 \%$ (from 0.47 to $0.38 \mathrm{mM}, P<0.001$ ) and little sulfate (from 0.029 to $0.033 \mathrm{mM}$ ) accumulated, suggesting an incomplete AVS oxidation (Figure S1B). Nitrate and sulfate levels of control sediment were too low to be detected $(<1 \mu \mathrm{M})$ while AVS was around $0.53 \mathrm{mM}$. On Day 8 , nitrate and nitrite levels barely changed but sulfate doubled from 0.04 to $0.09 \mathrm{mM}(P<0.001)$, which substantiated the AVS oxidation. Nitrate sediments on Day 16 featured a further nitrate reduction and coupled AVS oxidation, with a slight nitrate consumption (by 8.1\%, $P<0.001$ ), a significant AVS consumption (by $56.96 \%, P<0.001$ ) and a drastic sulfate accumulation (by $221 \%, P<0.001$ ).

This trend continued to the final stage (Day 16 - 32), except that Fe(II) participated in nitrate reduction. By the end of the incubation AVS was too depleted $(\sim 0.022 \mathrm{mM})$ to sustain nitrate reduction and instead a drastic consumption of ferrous ion took place (from 3.403 to $0.417 \mathrm{mM}$, by $87.75 \%$ ). Interestingly, the levels of total acid-soluble iron, i.e. the sum of $\mathrm{Fe}(\mathrm{II})$ and $\mathrm{Fe}(\mathrm{III})$, changed slightly ( $\sim 4.30 \%$ ) so that most $\mathrm{Fe}(\mathrm{II})$ was oxidized to Fe(III) (Figure S1D). Fe(II) consumption supported AVS depletion since Fe(III) spontaneously oxidizes AVS and thus Fe(III) accumulation indicated little AVS remaining. In summary, $0.450 \mathrm{mM}$ AVS and $2.99 \mathrm{mM} \mathrm{Fe}(\mathrm{II})$ were oxidized throughout the incubation, yielding $0.404 \mathrm{mM}$ sulfate and $2.559 \mathrm{mM} \mathrm{Fe}(\mathrm{III})$ respectively. Correspondingly, $2.51 \mathrm{mM}$ nitrate was reduced, yielding merely $0.094 \mathrm{mM}$ nitrite. Note that more nitrate in stoichiometry was consumed than the sum production of sulfate and $\mathrm{Fe}$ (III) if nitrate was denitrified to nitrogen gas.

\section{Nitrate input simplified community composition}

PCoA well substantiated the community dissimilarities between two groups (Figure 1A), where the nitrate group fell into distinct subgroups per sampling time but control samples showed no significant difference over time. For nitrate group, a longer incubation led to a larger dissimilarity between subgroups. This suggested that the notable community succession was in response to elevated nitrate but not to the onemonth incubation. We performed one-way ANOSIM to further compare the pairwise dissimilarities between subgroups with respect to dissimilarity significance $(P)$ and distribution (R2). For convenience, we used "C" to represent control sediments, and "N" to represent nitrate-amended sediments. For example, "4-C" refered to the control sediment on Day 4. Most subgroups were statistically different from each other except 4-C vs. 16-C and 8-C vs. 16-C (Figure 1B). Any pairwise dissimilarity between C-N and N-N subgroups was significant $(P<0.001)$, as PCoA suggested. In contrast, most control subgroups had comparable withinand between-subgroup dissimilarities (Figure 1C).

The relative abundance of categorized OTUs at the phylum or class level suggested a progressive dominance of beta-Proteobacteria in nitrate-amemded sediments, while classes Anaerolineae, delta-Proteobacteria and Euryarchaeota dominated the control (Figure 1D). The enrichment of beta-proteobacteria (from $1.79 \%$ to $61.81 \%, \sim 35$ folds) accounted for the dissimilarities among nitrate subgroups. Only $\sim 1 / 3$ of total 4990 OTUs could be assigned to a specific genus (Figure 1E). Among those, syntrophic bacteria (e.g. Smithella , Syntrophphorhabdus ,Syntrophphus and Syntrophobater ) and methanogenic archaea (e.g. Methanothrix , Methanoregula, Methanolinea, Methanobacterium and Methanomassiliicoccus ) dominated control and early nitrate communities. Compositions of control communities were rather time-steady so we only screened genera significantly changing over time within the nitrate group by paired T-test. We found that most genera were gradually outcompeted alongside the rise of genus Thiobacillus and Luteimonas (Figure 1F), and the core microbiomes of late nitrate subgroups (i.e., $16 \mathrm{~N}$ and $32 \mathrm{~N}$ ) greatly differed in taxonomy and abundance from control and the early nitrate subgroups. 


\section{Nitrate input simplified interactive network}

Ten networks based on Spearman's correlation coefficient were constructed to identify possible interactions. Key network topologies were listed in Table S1, from which we selected network size, modularity and connectivity to correlate three community metrics (Figure 2A, 2B, 2C). Sizes of control networks remained time-steady $(428.8 \pm 23.7$ nodes, $P>>0.05)$ while nitrate networks kept shrinking, from 308 (Day 0) to 94 nodes (Day 32), meaning that fewer OTU pairs were generating significant abundance covariations. Such changing patterns applied to all metrics in Figure 2 that elevated nitrate was simplifying community compositions (with respect to richness and $\alpha$-diversity) and microbial interactions. To visualize this simplification, all networks were plotted (Figure 3). The Fruchterman Reingold layout (Figure 3A) well exhibited the shrinking sizes of nitrate networks, while the Yifan Hu layout (Figure 3B) emphasized the decreasing modularity and node-node interactions.

Putative keystone nodes referred to those of great network connectivity, either within a module (i.e. module hub) or among modules (i.e. connector). Their taxa were therefore putative keystone taxa. We plotted the distribution of keystone nodes in Figure S2A. Of all the 3352 nodes, most were insignificant peripherals (97.67\%), 44 were module hubs and 34 were connectors (Table S2). No network hub was detected. When correlating node significance to OTU relative abundances (Figure S2B), we found that abundance did not necessarily determine its network significance, although keystone nodes turned to be sparse OTUs. Of the 78 keystone nodes, 31 were rare $(<0.1 \%$ relative abundance), 27 were moderately rare $(0.1-0.5 \%)$, 13 were moderately abundant $(0.5-1 \%)$, and only 7 were abundant $(>1 \%)$. In addition, the relative abundances of module hubs and connectors indicated no significant difference $(P>0.05)$.

To investigate the putative keystone taxa, we prepared a phylogenic tree that indicated (i) all the putative keystone taxa at the phylum or genus level; (ii) their phylogenic distance based on neighbor-joining method; and (iii) their keystone type (Figure S3). Most keystone nodes (85.5\%) could be assigned to a phylum but only $28.9 \%$ could be assigned to a genus. Members of phylum Proteobacteria (e.g. genusSmithella and Syntrophorhabdus ) and Chloroflexi(genus Bellilinea, Leptolinea and Pelolinea) constituted the majority of keystone taxa. Genus Smithella in particular, was found highly interactive in networks since 5 OTUs belonging to Smithella were keystone nodes. In addition, four keystone taxa were methanogenic archaea (Lee et al. 2015) and were members of genus Methanolinea, Methanothrix, and Methanobacterium.

Generally, the number of keystone nodes decreased as the nitrate amendment proceeded longer (Figure S4). That is, the network simplification occurred alongside the loss of keystone taxa. Recall that Thiobacillus was barely detectable in the control communities $(<0.02 \%$ relative abundance) but dominated the late nitrate communities ( $\sim 60.88 \%)$. However, it was never a keystone genus, showing no significant covariations in any network (Table S3).

\section{Predictive functional converge after nitrate input}

In FAPROTAX, most OTUs (> 90.7\%) failed to associate with either N- or S-metabolism. In the absence of favorable electron acceptors, control communities were rich in methanogens (Figure S5A), which were archaea in the phylum Euryarchaeota. The number of putative nitrate reducers (e.g., members of genus $N i$ trospira,Bacillus, Paracoccus) and sulfate reducers (e.g., members of genus Syntrophobacter, Desulfococcus ,Desulfobulbus, Desulfovibrio ) were comparable (Figure S5B, S5C). For nitrate communities, the number of putative nitrate reducers kept decreasing, which contradicted the drastic nitrate reduction possibly because the actual nitrate reducers were yet to be collected in the FAPROTAX database. Members of genus Thiobacillus ,Paracoccus, Sulfuricurvum or Acidithiobacillus were putative AVS oxidizers and Thiobacillus in particular, was assigned to dark-sulfide-oxidation and dark-oxidation-of-sulfur-compounds.

In PICRUSt, we focused on the changes of gene copy number based on a normalized PICRUSt table (KEGG Orthology Level 2 and 3). Each sample was rarefied to an equal sum of predicted gene copies so that the copy number of each functional item represented its strength. When analyzing the general metabolism (ko09100, Level 2), the late nitrate communities (16-N and 32-N) exhibited the greatest difference from the rest. Five orthologues (ko09102, 09104, 09106-09108) were enriched and four (ko09101, 09109-09111) were 
suppressed (Figure 4). Within ko09102 (energy metabolism), nitrogen metabolism had higher background abundances $(\sim 25000$ copies per community) than sulfur metabolism $(\sim 10500)$ and were more enriched after nitrate amendment (Figure S5E, S5F).

\section{Thiobacillus thiophilus became the best competitor in enriched communities}

The community of control sediments was serially diluted and cultured anaerobically in the NS media where nitrate was the sole electron acceptor and sulfide was the sole electron donor. This is to enrich bacteria having a complete or partial respiratory chain from sulfide, the initial e-donor to nitrate, the terminal eacceptor. The dominance of Thiobacillus reappeared in 20 out of 38 communities (Figure 5A) though it was initially sparse $(<1.3 \%$ relative abundance). This enrichment suggested the competent metabolic fitness of Thiobacillus . Linear regression also identified positive correlations between its relative abundance and nitrate consumption, nitrite production and sulfate production (Figure 5B-5D).

Community enrichments of different dilutabilities were streaked on NS and NTS plates (anaerobic at room temperature). However, no colony formed on the NS plates. Using solid NTS medium, 14 strains were isolated from all community enrichments and all were members of phylum Proteobacteria (Table 1). Unlike community enrichments, these pure isolates must a complete respiratory chain from thiosulfate to nitrate since interspecies cross-feeding was excluded. We then determined their metabolic characteristics using liquid NS and NTS medium, and found that strain S5643 (Thiobacillus thiophilus) was the sole sulfide oxidizer. PCR of selected functional genes involved in nitrogen and sulfur cycling found that (i) all strains except T83 (Sphingopyxis terrae) and T546 (Rhodanobacter lindaniclasticus ) had nitrate reductase narG or napA ; (ii) all strains had nitrite reductase nirK or nirS ; and (iii) thiosulfate oxidasesox B appeared only in 6 strains (Table 1), including S5643. These results confirmed the metabolic fitness of Thiobacillus, which was the sole sulfide-oxidizing denitrifier among the 14 isolates.

\section{Discussion}

Patterns of microbial community assembly and interaction in river sediments are far from clear, evidenced by the high proportion of uncultured and unclassified organisms present in subsurface environments together with the overwhelming discovery of new microbial lineages involved in major geochemical cycles (Anantharaman et al. 2016). In this study, we showed that nitrate amendment to sulfide-rich river sediments overwrote the redox conditions and thus simplified the microbial community composition and interaction by enriching sulfur-oxidizing denitrifiers.

Most denitrifiers are heterotrophs and a minor group are chemoautotrophs that utilize inorganic energy sources such as reduced sulfur and Fe(II) (Hayakawa et al. 2013). After nitrate amendment, we identified (i) a consistent nitrate reduction and the coupled AVS oxidation, (ii) an accumulation of nitrite and sulfate, and (iii) a drastic Fe(II) oxidation and the coupled Fe(III) accumulation in the final stage (Figure S6). Notably, more nitrate in stoichiometry was consumed than the sum consumption of AVS and Fe(II) if N2 was the end product (by denitrification), so there must have been cryptic electron donors fueling nitrate reduction (see supplementary information: the cryptic electron donor).

Among the identified genera, the control and early nitrate communities were dominated by methanogens (Patel \& Sprott 1990; Ma et al. 2005; Imachi et al. 2008; Braeuer et al. 2011; Kroeninger et al. 2017) and syntrophic bacteria (de Bok et al. 2001; McInerney et al. 2007; Qiu et al. 2008). When exposed to elevated nitrate, most genera were gradually outcompeted including some denitrifiers. Members of Thiobacillus were characterized as autotrophic sulfide oxidizers and denitrifiers (Bosch et al. 2012; Dolejs et al. 2015); T. ferrooxidans might even feed on Fe(II) (Nemati et al. 1998). The Thiobacillus detected from late nitrate communities shared the highest 16S rRNA gene similarity with T. thiophilus strain D24TN (99\%) and $T$. denitrificans strain NCIMB 9548 (98\%), both were obligate chemolithotrophs and facultative anaerobes, using thiosulfate or AVS as the electron donor, nitrate or oxygen as the electron acceptor, and $\mathrm{CO} 2$ as the sole carbon source (Kelly \& Wood 2000; Kellermann \& Griebler 2009). 
The dominance of Thiobacillus (in abundance) reappeared in the community enrichments (Figure 2E, 5A). Moreover, pure culture of isolated T. thiophilus was performing simultaneous nitrate-reduction, AVSoxidation and thiosulfate-oxidation (Table 1) so that it dominated NS enrichments (in abundance) because of its high metabolic fitness. Consistently, several studies identified an enhanced sulfide oxidation alongside the rise of Thiobacillus after nitrate amendment (Xu et al. 2014; He et al. 2017; He et al. 2018). The other winner Luteimonas was barely detectable at first but significantly enriched later, in agreement with a recent discovery in which Luteimonas dominated communities of sediment samples with an increasing proportion of recalcitrant carbon (Wu et al. 2018). Luteimonas spp. might be a potential Fe(II) oxidizer, as Zhang et al. identified a predicted ferric reductase gene and two subunits of ferredoxin nitrite reductase from the genome of L. abyssi XH301T (Zhang et al. 2015).

There are studies pointing out that community functionality is a direct reflection of physiochemical condition (Nelson et al. 2016; Gibbons 2017; Louca et al. 2017), and the rise of Luteimonas and Thiobacillus well substantiated that. Among so many denitrifiers, why lithotrophic Luteimonas and Thiobacillus rather than those organotrophs gained dominance remained unclear, under conditions of so abundant TOC.

Keystone taxa are believed important in maintaining the functionality and integrity of an ecosystem, whose extinction often leads to community fragmentation or even collapse (Wu et al. 2016). In this study, microbial interactive networks under nitrate amendment were getting smaller and simpler along with the loss of keystone taxa which predominantly comprised of members of Class Proteobacteria and Chloroflexi, as we hypothesized. We identified no strong correlation between OTU abundances and their interactive significance, although keystone nodes turned to be sparse OTUs. Instead, the network connectivity was speculated highly associated with microbial cross-feedings or metabolic interdependencies that were predominantly sustained by native auxotrophs (Mee et al. 2014; Embree et al. 2015; Ponomarova \& Patil 2015; Hubalek et al. 2017). For example, syntrophic bacteria (e.g. Smithella and Syntrophorhabdus) and methanogens (e.g. Methanolinea) were auxotrophs, performing extensive interspecies cross-feeding, and were keystone taxa in the microbial interactive networks.

Nitrate amendment might have other effects on microbial communities than simplification. We identified several rapid microbial responses to elevated nitrate, whereas the community richness, $\alpha$-diversity and evenness were little affected (Figure 2, Day 0 - 4). Several genera such as Methanomassiliicoccus, Vulcanibacillus, Thermomonas and Bacillus were significantly enriched on Day 4 but were eventually suppressed or eliminated (Figure 1E, 1F). So elevated nitrate introduced both days- and weeks- effects upon microbial communities of sulfide-rich river sediment. For the first few days it boosted biodiversity possibly by niche creation (e.g. bridging metabolic interdependencies between nitrate reducers and AVS oxidizers), in agreement with our previous work (Xu et al. 2014).

In summary, this study provides insights about the microbial assemblage and interactions in river sediments in the presence of elevated nitrate. Microbial communities were eventually simplified and specialized in nitrate reduction coupled with sulfide and $\mathrm{Fe}(\mathrm{II})$ oxidation. The significant loss of biodiversity and other functions were considered the negative ecological impacts of nitrate amendments because biodiversity increases community resistance to environmental perturbations (Griffiths \& Philippot 2013; Isbell et al. 2015; Evans et al. 2017; Xun et al. 2019). Thiobacillus and Luteimonas gained dominance while keystone taxa appeared to be diminished or extinct, leading to fragmentated interactive networks.

\section{Methods}

\section{Experiment design and sample process.}

in depth) from the Pearl River Delta $\left(22^{\circ} 14^{\prime} 50^{\prime \prime} \mathrm{N}, 113^{\circ} 11^{\prime} 50^{\prime \prime} \mathrm{E}\right)$ were sampled in December 2017. Plant residues, macrofauna and large particles were removed by hand grubbing. The resulting sediment was vigorously homogenized and distributed to $40 \mathrm{~g}$ (wet weight) per share. Sterilized pure water with or without $\sim 7.74 \mathrm{mM}$ calcium nitrate was added to the sediments, making each tube to the volume of $45 \mathrm{ml}$. In sum, we prepared 60 tubes with nitrate (as treatment) and 60 without nitrate (as control). All tubes were anaerobically cultured at room temperature. 12 treatment and 12 control tubes were collected on Day $0,4,8,16$, 
32 respectively for physiochemical characterizations and DNA extraction. We quantified the levels of AVS, ferrous iron, total iron, $\mathrm{pH}$, nitrate, nitrate, sulfate, and TOC (total organic carbon). See supplementary information for measurements. For convenience, we assigned all 120 samples into two groups: nitrate and control, 60 samples each. Each group had five subgroups based on sampling time, i.e. 0-N, 4-N, 8-N, 16-N, and 32-N for nitrate group, while 0-C, 4-C, 8-C, 16-C and 32-C for control. Each subgroup consisted of 12 replicates.

To analyze the composition of nitrate-reducing sulfur-oxidizing bacteria (NR-SOB) in the sediment, a groups of serial dilution enrichments (Lagier et al. 2018) with 12 series repetitions were prepared in medium NS (nitrate as the sole electron acceptor and sulfide as the sole electron donor). See supplementary information for medium chemical compositions. $1 \mathrm{~g}$ wet sediment ( $\sim 80 \% \mathrm{w} / \mathrm{w}$ water content) was added to $9 \mathrm{ml}$ medium to get the 101 dilution factor. The serial dilution was conducted every ten-fold until 10-8. Enrichments at each dilution factor had 12 parallels. Note that parallels here were not equivalent to replicates since every dilution was random so that the resulting community compositions might differ. See supplementary information for sample cultivation. All samples were quantified of remaining nitrate and sulfate to estimate the metabolic intensity, which was roughly represented by the amount of nitrate consumption and sulfide/thiosulfate consumption. The ultimate NR-SOB strains were isolated by plate-streaking on medium NTS (thiosulfate as the sole electron donor) plates supplemented with $1.5 \%$ agar respectively. After one-month anaerobic cultivation, different colonies were isolated, purified and identified by colony PCR of full length 16S rRNA gene.

\section{Illumina Miseq sequencing and predictive functional profiling.}

Sequencing of $16 \mathrm{~S}$ ribosome RNA gene V4 region was used to track community successions. Qualified DNA libraries were sequenced and clustered into de novo OTUs by the Usearch (v9.2.64) pipeline (Edgar 2010). See supplementary information for detail. Briefly, each sample was rarefied to 10,000 clean reads. Sequencing of 16S rRNA gene gives no direct metabolic information so we used FAPROTAX (Loucaet al. 2016) and PICRUSt (Langille et al. 2013) just to roughly estimate the functional changes under nitrate stimulation. Clean 16S rRNA gene tags were re-clustered against the GreenGenes database (v13.8, 97_otus.fasta) to be compatible with FAPROTAX (v1.1) and PICRUSt (v1.1.1). FAPROTAX was used to identify putative functional microbial members, and PICRUSt was to compare the gene abundance before and after nitrate stimulation, especially those involved in $\mathrm{N}$ or $\mathrm{S}$ metabolisms. Both predictions were based on the same normalized closed-reference OTU table.

\section{Interactive network construction and classification.}

Each network was constructed based on Spearman's correlation of the 12 replicates by the MENA pipeline (http: //ieg4. rccc. ou. edu/MENA/) (Zhou et al. 2010; Zhou et al. 2011; Deng et al.2012) yielding 10 networks. In network construction, the threshold was automatically determined in the RMT-based modeling. Only OTUs present in more than 8 out of 12 replicates were involved. Output networks were visualized using Gephi (v0.9.2). A visualized interactive network consisted of nodes and links (or edges). A node represented an OTU that had significant Spearman's correlations (above the network threshold) with other nodes. A link between two nodes represented their negative or positive covariation (Montoya et al. 2006; Bascompte 2007) depend on the calculated correlation coefficient (-1 to +1). A positive correlation meant the two nodes were sharing a same changing pattern. A module referred to a group of nodes that were highly interconnected within the group and had fewer or no connections outside the group. MENA has four built-in methods for module separation, and we selected the greedy modularity optimization because it generated the highest

Modularity index $(M)$. Generally, the number of nodes defines the network size, while the numbers of modules and links per node define the network complexity. See supplementary information for visualization settings.

\section{Statistical analysis.}

Shannon's index $(\mathrm{H})$ and Simpson's evenness $(\mathrm{J})$ were calculated to represent alpha-diversity of each community. Community dissimilarity between control and nitrate group was compared using PCoA and one-way ANOSIM, both were based on Brey-Curtis distance. Genera that significantly changed in relative abundance 
over time or over treatment were selected. We confirmed the compliance of normal distribution of genus frequencies and then quantified the dissimilarities by one-way T-test. Alpha-diversity, PCoA and ANOSIM were performed with R (Vegan package, v2.4-6) (Oksanen et al. 2010).

\section{Acknowledgement}

This work was funded by:

- National Natural Science Foundation of China (91851202, 51678163, U1701243)

- GDAS' Special Project of Science and Technology Development (2019GDASYL-0104005)

- Guangdong Provincial Programs for Science and Technology Development (2018B030324002, 2019B110205004)

- Science and Technology Project of Guangzhou (201707020021).

\section{Reference}

1.

Anantharaman, K., Brown, C.T., Hug, L.A., Sharon, I., Castelle, C.J., Probst, A.J. et al. (2016). Thousands of microbial genomes shed light on interconnected biogeochemical processes in an aquifer system. Nature Communications , 7 .

2 .

Bascompte, J. (2007). Networks in ecology. Basic and Applied Ecology , 8, 485-490.

3 .

Bosch, J., Lee, K.-Y., Jordan, G., Kim, K.-W. \& Meckenstock, R.U. (2012). Anaerobic, nitrate-dependent oxidation of pyrite nanoparticles by Thiobacillus denitrificans. Environmental science $\&$ technology , 46, 2095-2101.

4 .

Braeuer, S.L., Cadillo-Quiroz, H., Ward, R.J., Yavitt, J.B. \& Zinder, S.H. (2011). Methanoregula boonei gen. nov., sp. nov., an acidiphilic methanogen isolated from an acidic peat bog. International Journal of Systematic and Evolutionary Microbiology, 61, 45-52.

5 .

Brunet, R.C. \& GarciaGil, L.J. (1996). Sulfide-induced dissimilatory nitrate reduction to ammonia in anaerobic freshwater sediments.Fems Microbiology Ecology, 21, 131-138.

6.

Burgin, A.J. \& Hamilton, S.K. (2007). Have we overemphasized the role of denitrification in aquatic ecosystems? A review of nitrate removal pathways. Frontiers in Ecology and the Environment , 5, 89-96.

7.

Burgin, A.J. \& Hamilton, S.K. (2008). NO(3)(-)-driven SO(4)(2-) production in freshwater ecosystems: Implications for N and S cycling.Ecosystems , 11, 908-922.

8.

Chen, M., Tang, H., Ma, H., Holland, T.C., Ng, K.Y.S. \& Salley, S.O. (2011). Effect of nutrients on growth and lipid accumulation in the green algae Dunaliella tertiolecta. Bioresource technology, 102, 1649-1655.

9.

Cordero, O.X. \& Datta, M.S. (2016). Microbial interactions and community assembly at microscales. Current opinion in microbiology , 31, 227-234.

10. 
de Bok, F.A.M., Stams, A.J.M., Dijkema, C. \& Boone, D.R. (2001). Pathway of propionate oxidation by a syntrophic culture of Smithella propionica and Methanospirillum hungatei. Applied and Environmental Microbiology , 67, 1800-1804.

11.

Deng, Y., Jiang, Y.-H., Yang, Y., He, Z., Luo, F. \& Zhou, J. (2012). Molecular ecological network analyses. Bmc Bioinformatics , 13.

12 .

Dolejs, P., Paclík, L., Maca, J., Pokorna, D., Zabranska, J. \& Bartacek, J. (2015). Effect of S/N ratio on sulfide removal by autotrophic denitrification. Applied microbiology and biotechnology, 99, 2383-2392.

13.

Donaldson, G.P., Lee, S.M. \& Mazmanian, S.K. (2016). Gut biogeography of the bacterial microbiota. Nature Reviews Microbiology , 14, 20-32.

14.

Edgar, R.C. (2010). Search and clustering orders of magnitude faster than BLAST. Bioinformatics , 26, 2460-2461.

15.

Embree, M., Liu, J.K., Al-Bassam, M.M. \& Zengler, K. (2015). Networks of energetic and metabolic interactions define dynamics in microbial communities. Proceedings of the National Academy of Sciences of the United States of America, 112, 15450-15455.

16.

Evans, S., Martiny, J.B. \& Allison, S.D. (2017). Effects of dispersal and selection on stochastic assembly in microbial communities. The ISME journal , 11, 176.

17.

Fowler, D., Coyle, M., Skiba, U., Sutton, M.A., Cape, J.N., Reis, S.et al. (2013). The global nitrogen cycle in the twenty-first century. Philosophical Transactions of the Royal Society B-Biological Sciences , 368.

18.

Gibbons, S.M. (2017). MICROBIAL COMMUNITY ECOLOGY Function over phylogeny. Nature Ecology E Evolution, 1.

19.

Griffiths, B.S. \& Philippot, L. (2013). Insights into the resistance and resilience of the soil microbial community. FEMS microbiology reviews, 37, 112-129.

20.

Hayakawa, A., Hatakeyama, M., Asano, R., Ishikawa, Y. \& Hidaka, S. (2013). Nitrate reduction coupled with pyrite oxidation in the surface sediments of a sulfide-rich ecosystem. Journal of Geophysical ResearchBiogeosciences , 118, 639-649.

21.

He, Z., Huang, R., Liang, Y., Yu, G., Chong, Y. \& Wang, L. (2018). Index for nitrate dosage calculation on sediment odor control using nitrate-dependent ferrous and sulfide oxidation interactions. Journal of environmental management , 226, 289-297.

22. 
He, Z., Long, X., Li, L., Yu, G., Chong, Y., Xing, W. et al.(2017). Temperature response of sulfide/ferrous oxidation and microbial community in anoxic sediments treated with calcium nitrate addition. Journal of environmental management, 191, 209-218.

23.

Hubalek, V., Buck, M., Tan, B., Foght, J., Wendeberg, A., Berry, D.et al. (2017). Vitamin and Amino Acid Auxotrophy in Anaerobic Consortia Operating under Methanogenic Conditions. Msystems , 2.

24.

Imachi, H., Sakai, S., Sekiguchi, Y., Hanada, S., Kamagata, Y., Ohashi, A. et al. (2008). Methanolinea tarda gen. nov., sp nov., a methane-producing archaeon isolated from a methanogenic digester sludge.International Journal of Systematic and Evolutionary Microbiology , 58, 294-301.

25.

Isbell, F., Craven, D., Connolly, J., Loreau, M., Schmid, B., Beierkuhnlein, C. et al. (2015). Biodiversity increases the resistance of ecosystem productivity to climate extremes. Nature, 526, 574 .

26.

Kellermann, C. \& Griebler, C. (2009). Thiobacillus thiophilus sp nov., a chemolithoautotrophic, thiosulfateoxidizing bacterium isolated from contaminated aquifer sediments. International Journal of Systematic and Evolutionary Microbiology, 59, 583-588.

27.

Kelly, D.P. \& Wood, A.P. (2000). Confirmation of Thiobacillus denitrificans as a species of the genus Thiobacillus, in the beta-subclass of the Proteobacteria, with strain NCIMB 9548 as the type strain. International Journal of Systematic and Evolutionary Microbiology, 50, 547-550.

28.

Kroeninger, L., Gottschling, J. \& Deppenmeier, U. (2017). Growth Characteristics of Methanomassiliicoccus luminyensis and Expression of Methyltransferase Encoding Genes. Archaea-an International Microbiological Journal .

29.

Lagier, J.-C., Dubourg, G., Million, M., Cadoret, F., Bilen, M., Fenollar, F. et al. (2018). Culturing the human microbiota and culturomics'. Nature Reviews Microbiology, 16, 540-550.

30.

Lagier, J.-C., Khelaifia, S., Alou, M.T., Ndongo, S., Dione, N., Hugon, P. et al. (2016). Culture of previously uncultured members of the human gut microbiota by culturomics. Nature microbiology , 1, 16203.

31.

Lagier, J.C., Armougom, F., Million, M., Hugon, P., Pagnier, I., Robert, C. et al. (2012). Microbial culturomics: paradigm shift in the human gut microbiome study. Clinical Microbiology and Infection , 18, 1185-1193.

32 .

Langille, M.G.I., Zaneveld, J., Caporaso, J.G., McDonald, D., Knights, D., Reyes, J.A. et al. (2013). Predictive functional profiling of microbial communities using $16 \mathrm{~S}$ rRNA marker gene sequences. Nature Biotechnology , 31, 814-+.

33. 
Lee, S.-H., Park, J.-H., Kim, S.-H., Yu, B.J., Yoon, J.-J. \& Park, H.-D. (2015). Evidence of syntrophic acetate oxidation by Spirochaetes during anaerobic methane production. Bioresource technology, 190, 543-549.

34.

Louca, S., Jacques, S.M.S., Pires, A.P.F., Leal, J.S., Srivastava, D.S., Parfrey, L.W. et al. (2017). High taxonomic variability despite stable functional structure across microbial communities. Nature Ecology $\mathcal{E}$ Evolution, 1 .

35.

Louca, S., Parfrey, L.W. \& Doebeli, M. (2016). Decoupling function and taxonomy in the global ocean microbiome. Science, 353, 1272-1277.

36.

Lutz, W., Sanderson, W. \& Scherbov, S. (2001). The end of world population growth. Nature , 412, 543-545. 37.

Ma, K., Liu, X.L. \& Dong, X.Z. (2005). Methanobacterium beijingense sp nov., a. novel methanogen isolated from anaerobic digesters. International Journal of Systematic and Evolutionary Microbiology, 55, 325-329.

38.

McInerney, M.J., Rohlin, L., Mouttaki, H., Kim, U., Krupp, R.S., Rios-Hernandez, L. et al. (2007). The genome of Syntrophus aciditrophicus: Life at the thermodynamic limit of microbial growth.Proceedings of the National Academy of Sciences of the United States of America, 104, 7600-7605.

39.

McIsaac, G.F., David, M.B., Gertner, G.Z. \& Goolsby, D.A. (2001). Eutrophication: nitrate flux in the Mississippi River. Nature, 414, 166.

40.

Mee, M.T., Collins, J.J., Church, G.M. \& Wang, H.H. (2014). Syntrophic exchange in synthetic microbial communities. Proceedings of the National Academy of Sciences of the United States of America, 111, E2149E2156.

41.

Montoya, J.M., Pimm, S.L. \& Solé, R.V. (2006). Ecological networks and their fragility. Nature, 442, 259.

42.

Nelson, M.B., Martiny, A.C. \& Martiny, J.B.H. (2016). Global biogeography of microbial nitrogen-cycling traits in soil.Proceedings of the National Academy of Sciences of the United States of America, 113, 80338040 .

43.

Nemati, M., Harrison, S.T.L., Hansford, G.S. \& Webb, C. (1998). Biological oxidation of ferrous sulphate by Thiobacillus ferrooxidans: a review on the kinetic aspects. Biochemical Engineering Journal , 1, 171-190. 44.

Oksanen, J., Blanchet, F.G., Kindt, R., Legendre, P., O'hara, R.B., Simpson, G.L. et al. (2010). Vegan: community ecology package. R package version 1.17-4. http://cran. r-project. org $>$. Acesso em , 23, 2010.

45. 
Patel, G.B. \& Sprott, G.D. (1990). METHANOSAETA-CONCILII GEN-NOV, SP-NOV (METHANOTHRIXCONCILII) AND METHANOSAETA-THERMOACETOPHILA NOM-REV, COMB-NOV. International Journal of Systematic Bacteriology , 40, 79-82.

46.

Ponomarova, O. \& Patil, K.R. (2015). Metabolic interactions in microbial communities: untangling the Gordian knot. Current opinion in microbiology , 27, 37-44.

47.

Qiu, Y.-L., Hanada, S., Ohashi, A., Harada, H., Kamagata, Y. \& Sekiguchi, Y. (2008). Syntrophorhabdus aromaticivorans gen. nov., sp. nov., the first cultured anaerobe capable of degrading phenol to acetate in obligate syntrophic associations with a hydrogenotrophic methanogen. Applied and Environmental Microbiology , 74, 2051-2058.

48.

Wu, L., Yang, Y., Chen, S., Zhao, M., Zhu, Z., Yang, S. et al.(2016). Long-term successional dynamics of microbial association networks in anaerobic digestion processes. Water Research , 104, 1-10.

49.

Wu, X., Wu, L., Liu, Y., Zhang, P., Li, Q., Zhou, J. et al.(2018). Microbial interactions with dissolved organic matter drive carbon dynamics and community succession. Frontiers in microbiology, 9.

50.

Xia, X., Zhang, S., Li, S., Zhang, L., Wang, G., Zhang, L. et al.(2018). The cycle of nitrogen in river systems: sources, transformation, and flux. Environmental Science-Processes $\&$ Impacts , 20, 863-891.

51.

Xu, M., Zhang, Q., Xia, C., Zhong, Y., Sun, G., Guo, J. et al.(2014). Elevated nitrate enriches microbial functional genes for potential bioremediation of complexly contaminated sediments. The ISME journal , 8, 1932 .

52.

Xun, W., Li, W., Xiong, W., Ren, Y., Liu, Y., Miao, Y. et al.(2019). Diversity-triggered deterministic bacterial assembly constrains community functions. Nature communications , 10.

53.

Zehavi, T., Probst, M. \& Mizrahi, I. (2018). Insights into culturomics of the rumen microbiome. Frontiers in microbiology, 9 .

54.

Zhang, L., Wang, X., Yu, M., Qiao, Y. \& Zhang, X.-H. (2015). Genomic analysis of Luteimonas abyssi XH031(T): insights into its adaption to the subseafloor environment of South Pacific Gyre and ecological role in biogeochemical cycle. Bmc Genomics , 16.

55.

Zhou, J., Deng, Y., Luo, F., He, Z., Tu, Q. \& Zhi, X. (2010). Functional Molecular Ecological Networks. Mbio , 1 .

56.

Zhou, J., Deng, Y., Luo, F., He, Z. \& Yang, Y. (2011). Phylogenetic Molecular Ecological Network of Soil Microbial Communities in Response to Elevated CO2. Mbio , 2. 


\section{Figures}

Figure 1. Metrics concerning community successions. In the PCoA plot based on Bray-Curtis distance (A ), control communities were rather time-steady but those of nitrate group were progressively drifting from previous states. Samples falling out of the circle were too divergent from other replicates and were excluded from futher analysis. Communities of all subgroups except the pairs of 4-C/16-C and 8-C/16-C were significantly different $(P<0.05)$ in the significance matrix of one-way ANOSIM $(\mathbf{B})$, especially those in the N-N and $\mathrm{C}-\mathrm{N}$ areas $(P<0.001)$. Most control communities had comparable dissimilarities within and among subgroups, while dissimilarities among nitrate subgroups were much greater than dissimilarities within nitrate subgroup $(\mathbf{C})$. The categorized taxonomic composition at the phylum and class levels suggested a stepwise enrichment of beta-proteobacteria occuring to nitrate sediment communities (D ). In particular, genera Thiobacillus andLuteimonas outcompeted other genera (E , unclassified genera were left blank) and became dominant $(\mathbf{F})$.

Figure 2. Comparison of community and network metrics.(A ) Number of OTUs present in more than 8 out of 12 sediment replicates for each subgroup, (B ) Shannon's diversity, (C) Simpson's evenness, (D ) Number of nodes of each constructed network per subgroup, (E ) Number of modules and (F ) Number of links. When referring to network modularity and node connectivity, we focused on modules consisting of more than 5 interconnected nodes. (A ), (B ) and (C) were calculated based on bootstrap method $(\mathrm{N}=1000)$. R2 and $\mathrm{F}$-significance $(P)$ were calculated by regression analyses.

Figure 3. Networks based on 12 replicates were constructed by the RMT-MENA program. Fruchterman Reingold layout (A ) exhibited the shrinking nitrate networks. Node size was proportional to its degree. Modules consisting of over 5 nodes were randomly colored, the rest left grey. Positive covariations were colored green and negative covariations were colored red. For convenience, we unified the node density so that network sizes could be directly read based on the diameter. Yifan Hu layout (B ) emphasized the network connectivity. Nodes and links of the same module shared the same color, and all nodes were in a uniform size. In summary, Nitrate networks were getting smaller (A) and simpler (B).

Figure 4. Predictive metabolic profiling, based on KEGG orthology (ko) Level 2. The late nitrate communities (16-N and 32-N) were significantly different from the others, as seen from the enhanced metabolisms of rare amino acids, glycan, nucleotide, cofactors and vitamins, and seen from the suppressed expression in terpenoids, polyketides, xenobiotics, lipid and carbohydrate.

Figure 5. Thiobacillus became the best competitor in diluted communities. (A) community compositions of diluted, enriched communities at the genus level. CK - control communities; NS - the medium, followed by the dilution factors. (B), (C) and (D) - linear fitting for Thiobacillusrelative abundance versus nitrate consumption, nitrite production and sulfate production.

Table 1. Strains isolated from enriched sediment communities on NTS plates.

\begin{tabular}{llllll}
\hline Strain & Closest relative & Identity & Class of Proteobacteria & Nitrate reduction & Sulfide oxidat \\
\hline F21 & Ciceribacter thiooxidans & $99.9 \%$ & $\alpha$ & $(+)$ & $(-)$ \\
T6647 & Aminobacter niigataensis & $99.3 \%$ & $\alpha$ & $(+)$ & $(-)$ \\
T677 & Afipia felis & $97.4 \%$ & $\alpha$ & $(+)$ & $(-)$ \\
T681 & Bosea robiniae & $98.9 \%$ & $\alpha$ & $(+)$ & $(-)$ \\
T83 & Sphingopyxis terrae & $99.8 \%$ & $\alpha$ & $(+)$ & $(-)$ \\
T5712 & Achromobacter insuavis & $99.4 \%$ & $\beta$ & $(+)$ & $(-)$ \\
S5643 & Thiobacillus thiophilus & $98.4 \%$ & $\beta$ & $(+)$ & $(+)$ \\
S544 & Azonexus caeni & $99.5 \%$ & $\beta$ & $(+)$ & $(-)$ \\
T84 & Hydrogenophaga intermedia & $99.0 \%$ & $\beta$ & $(+)$ & $(-)$ \\
T572 & Alicycliphilus denitrificans & $99.6 \%$ & $\beta$ & $(+)$ & $(-)$ \\
T763 & Pseudomonas stutzeri & $99.2 \%$ & $\gamma$ & $(+)$ & $(-)$
\end{tabular}




\begin{tabular}{llllll}
\hline Strain & Closest relative & Identity & Class of Proteobacteria & Nitrate reduction & Sulfide oxidat \\
\hline S685 & Pseudoxanthomonas mexicana & $99.4 \%$ & $\gamma$ & $(+)$ & $(-)$ \\
T546 & Rhodanobacter lindaniclasticus & $99.6 \%$ & $\gamma$ & $(+)$ & $(-)$ \\
\hline
\end{tabular}

